\title{
Decision-Making and Hospital Care
}

\section{POLST FORMS WITHIN AN ACUTE CARE SETTING}

R Fullam 1 , K Detering 1 , W Silvester ${ }^{1}{ }^{1}$ Respecting Patient Choices

There are only a limited number of studies on the content and completion of Physician Orders for Life Sustaining Treatment (POLST) forms. No study has specifically examined the use of POLST forms within an acute hospital setting. We audited 1096 randomly selected Resuscitation Plans, a locally developed form of POLST, completed in a university hospital during 2011. Matching patient identification numbers resulted in 789 individual patients (49.7\% Male; 64\% aged 75+), of whom 187 had multiple plans during the audit period. The most recent plan for each patient was examined for content. Plans were

most commonly completed by Registrars $(539,68.3 \%)$, and $99.7 \%$ of plans were signed. The majority of plans indicated orders for treatment limitation $(608,77.1 \%)$, and these patients were significantly older than patients with an order for full treatment $(\mathrm{p}=.001)$. Information on the decision making process was completed in 540 cases (68.4\%). Of these, $63 \%$ had evidence that the patient/family had been involved in, or were informed of the decision. There was a significant association between limitation of treatment and evidence of family/ patient involvement in the decision making process ( $p=.001)$. Forty-nine percent of patients with multiple plans had orders in the most recent plan for less-aggressive treatment, compared to the prior plan. A further $44 \%$ had no changes to treatment orders over consecutive plans.

The use of POLST forms in an acute care environment is dynamic and complex. Evidence of patient/family involvement in the decision making process should increase as local advance care planning programs progress. 